\title{
Connective Tissue Growth Factor Promotes Fibrosis Downstream of TGF $\beta$ and IL-6 in Chronic Cardiac Allograft Rejection
}

\author{
A. J. Booth ${ }^{a}$, K. Csencsits-Smith ${ }^{c}$, S. C. Wood ${ }^{b}$, \\ G. Lu ${ }^{b}$, K. E. Lipson ${ }^{d}$ and D. K. Bishop ${ }^{a, b, *}$ \\ ${ }^{a}$ Graduate Program in Immunology and ${ }^{\mathrm{b}}$ Department of \\ Surgery, University of Michigan Medical Center, \\ Ann Arbor, MI \\ ${ }^{c}$ Department of Pathology and Laboratory Medicine, \\ University of Texas Health Science Center at Houston, \\ Houston, TX \\ dFibroGen Inc., San Francisco, CA \\ * Corresponding author: D. Keith Bishop, \\ kbishop@umich.edu
}

Cardiac transplantation is an effective treatment for multiple types of heart failure refractive to therapy. Although immunosuppressive therapeutics have increased survival rates within the first year posttransplant, chronic rejection (CR) remains a significant barrier to long-term graft survival. Indicators of CR include patchy interstitial fibrosis, vascular occlusion and progressive loss of graft function. Multiple factors have been implicated in the onset and progression of CR, including TGF $\beta$, IL- 6 and connective tissue growth factor (CTGF). While associated with CR, the role of CTGF in CR and the factors necessary for CTGF induction in vivo are not understood. To this end, we utilized forced expression and neutralizing antibody approaches. Transduction of allografts with CTGF significantly increased fibrotic tissue development, though not to levels observed with TGF $\beta$ transduction. Further, intragraft CTGF expression was inhibited by IL6 neutralization whereas TGF $\beta$ expression remained unchanged, indicating that IL- 6 effects may potentiate TGF $\beta$-mediated induction of CTGF. Finally, neutralizing CTGF significantly reduced graft fibrosis without reducing TGF $\beta$ and IL-6 expression levels. These findings indicate that CTGF functions as a downstream mediator of fibrosis in CR, and that CTGF neutralization may ameliorate fibrosis and hypertrophy associated with CR.

Key words: Chronic rejection, CTGF, fibrosis, IL-6, TGF $\beta$

Received 29 June 2009, revised 03 August 2009 and accepted for publication 04 August 2009

\section{Introduction}

Chronic rejection (CR) is a significant barrier to long-term graft acceptance. Manifestations of CR include interstitial fibrosis, occlusion of luminal structures and progressive loss of graft function (1-7). The etiology of CR is not fully understood. However, multiple factors have been associated with its onset and progression, especially TGF $\beta$. TGF $\beta$ overexpression is linked with $\mathrm{CR}(8,9)$, and may negatively impact graft survival through chemotactic and profibrotic effects (10). However, in addition to its deleterious fibrotic effects on the graft, TGF $\beta$ 's immunosuppressive and antiproliferative functions may be indispensable for graft and host survival (11). For example, TGF $\beta$ plays a critical role in the induction and function of $T$ regulatory cells (Treg), which are believed to contribute to graft acceptance (1214). Further, TGF $\beta$ inhibits T- and B-cell proliferation (10) and represses cancers of epithelial cell origin (15). These opposing effects make TGF $\beta$ a suboptimal target for CR treatments and have prompted investigation into the downstream mediators of TGF $\beta$ in CR pathology. Identifying downstream mediators of CR may facilitate the development of therapeutics that negate the fibrosis-inducing activity of TGF $\beta$ while sparing its antiinflammatory and antiproliferative effects.

One such downstream mediator, known to be induced by TGF $\beta$ in multiple cell types (16), including cardiac myocytes and fibroblasts (17), is connective tissue growth factor (CTGF). CTGF plays an important role in the development of connective tissue as well as the formation of scar tissue $(18,19)$, and is upregulated in multiple fibrotic disorders, including CR of cardiac and kidney grafts $(8,20-$ 22). CTGF mediates multiple profibrotic effects ascribed to TGF $\beta$ including increased extracellular matrix production, fibroblast proliferation and enhancement of adhesive responses (22). Thus, as CTGF is induced by TGF $\beta$ and because CTGF mediates profibrotic effects, CTGF has been proposed as a therapeutic target for limiting the deleterious fibrotic effects of TGF $\beta$ while sparing its immunemodulatory functions $(8,23,24)$.

CTGF induction by TGF $\beta$ has been observed in settings of cardiac fibrosis (22). However, we have previously reported that transduction of syngeneic grafts with TGF $\beta$ is insufficient to induce CTGF or CR (8). Hence, TGF $\beta$-mediated 
induction of CTGF in vivo is contextually dependent. One such contextual difference between allogeneic and syngeneic grafts is the development of alloimmune responses which may provide factors that crosstalk with TGF $\beta$ signaling (25). This prompted further investigation into immune parameters that potentiated TGF $\beta$-induced fibrosis and led to the identification of a critical role in the initiation and progression of CR for IL-6 (26), a cytokine that modulates the effects of TGF $\beta$ in multiple cell types (27-29).

Because TGF $\beta$, CTGF and IL-6 have established associations with $\mathrm{CR}(8,26)$, we investigated the relationships between these cytokines utilizing overexpression and neutralization approaches. These findings support the role of CTGF as a promoter of cardiac graft fibrosis and indicate that it functions downstream of TGF $\beta$ and IL-6. Further, these findings indicate that CTGF neutralization holds promise as a therapeutic approach for limiting the fibrosis associated with CR.

\section{Materials and Methods}

\section{Mice}

Female C57BL/6 $\left(\mathrm{H}-2^{b}\right)$ and BALB/c $\left(\mathrm{H}-2^{d}\right)$ mice were obtained from Charles River Laboratories (Raleigh, NC) and were kept under microisolator conditions. The use of mice for these studies was reviewed and approved by the University of Michigan's Committee on the Use and Care of Animals.

\section{Vascularized cardiac transplantation}

Heterotopic cardiac transplantation was performed as described (30). Briefly, the aorta and pulmonary artery of the donor heart were anastomosed end-to-side to the recipient's abdominal aorta and inferior vena cava, respectively. On perfusion with the recipient's blood, the transplanted heart resumes contraction. Graft function is monitored by abdominal palpation.

\section{In vivo mAb therapy}

Anti-CD4 (hybridoma GK1.5, obtained from American Type Culture Collection, Manassas, VA), anti-CD40L (hybridoma MR1, kindly provided by Dr. Randy Noelle, Dartmouth College) and anti-IL-6 (hybridoma MP5-20F3, obtained from American Type Culture Collection, Manassas VA, with permission of DNAX) mAbs were prepared by Bio X Cell (West Lebanon, NH). Allograft recipients were transiently depleted of $C D 4+$ cells by i.p. injection of $1 \mathrm{mg}$ of anti-CD4 mAb on days $-1,0$ and 7 posttransplant $(8,26)$. For inductive anti-CD40L therapy, allograft recipients were injected i.p. with 1 mg of anti-CD40L on days 0,1 and 2 posttransplant $(8,26)$. Anti-IL-6 mAb or control rat IgG (Sigma, St. Louis, MO) was administered by i.p. injection of $1 \mathrm{mg}$ on days $-1,1$ and 3 and weekly thereafter $(26,31)$. Allograft recipients treated with anti-CTGF mAb (FG-3019, kindly provided by FibroGen Inc., San Francisco, CA $(32,33)$ ) or control human IgG (Sigma) received $0.5 \mathrm{mg}$ i.p. twice weekly beginning on day 7 posttransplant.

\section{Adenoviral-mediated transduction of cardiac grafts}

Transduction was performed as previously described $(8,34,35)$. Briefly, cardiac grafts were perfused via the aorta with $5 \times 10^{8}$ pfu of E1/E3 deleted adenoviral vectors encoding the active form of human TGF $\beta 1$ (AdTGF $\beta$ ) (8,34), human CTGF (AdCTGF) (36) or beta-galactosidase (Adßgal) $(8,34,35)$. Following perfusion, donor grafts were placed in iced Ringer's solution for $1 \mathrm{~h}$ prior to transplantation. Previous studies with Ad $\beta$ gal have revealed a patchy distribution of transgene expression by both cardiac and vascular cells that persists for at least 8 weeks posttransplant (35).
Morphometric analysis of cardiac graft fibrosis and hypertrophy Graft fibrosis was quantified by morphometric analysis of Masson's trichrome-stained sections using iPLab software (Scanalytics Inc., Fairfax, VA). Mean fibrotic area was calculated from 10 to 12 areas per heart section analyzed at $200 \times$ magnification $(26,37)$. To quantify cardiomyocyte area as a measure of hypertrophy, digital outlines were drawn around at least 80 cardiomyocytes from views of $\mathrm{H} \& \mathrm{E}$-stained sections at $200 \times$ magnification. Areas within outlines were quantified using SCION IMAGE Beta 4.0.2 software (Scion Corporation, Frederick, MD) to measure cardiomyocyte cell size (38). A minimum of eight hearts were analyzed per group for both analysis techniques.

\section{Quantitative real-time PCR}

Graft RNA was isolated by homogenizing tissues in TRIzol reagent (Invitrogen, Carlsbad, CA) as per manufacturer's protocol. Five micrograms of total RNA were reverse transcribed using Oligo dT, dNTPS, MMLVRT (Invitrogen), RNAsin (Promega, Madison, WI) in PCR buffer (Roche, Indianapolis, IN). Resulting cDNA was purified by a $1: 1$ extraction with phenol/chloroform/isoamyl (25:24:1) then precipitated in one volume $3 \mathrm{M}$ $\mathrm{NaOAc}$ and two volumes absolute ethanol. Levels of atrial natriuretic peptide (ANP), CTGF, IL-6, TGF $\beta, I L-17$ and T-cell receptor $\beta$ constant region (TCR $\beta$ ) message were determined by quantitative real-time PCR using iQ SYBR master mix (Bio-Rad, Hercules, CA) in a Rotor-Gene 3000 thermocycler (Corbett Life Science, San Francisco, CA). Expression levels were determined relative to GAPDH using the Rotor-Gene comparative concentration utility.

Primer sequences were as follows:

ANP (Nppa) forward 5'-GGAGGTCAACCCACCTCTG-3'

ANP (Nppa) reverse 5'-GCTCCAATCCTGTCAATCCTAC-3'

CTGF (Ctgf) forward 5'-GGAAAACATTAAGAAGGGCAAAA-3'

CTGF (Ctgf) reverse 5'-CCGCAGAACTTAGCCCTGTA-3'

GAPDH (Gapdh) forward 5'-CTGGTGCTGAGTATGTCGTG-3'

GAPDH (Gapdh) reverse 5'-CAGTCTTCTGAGTGGCAGTG-3'

IL-6 (//6) forward 5'-CGTGGAAATGAGAAAAGAGTTGT-3'

IL-6 (//6) reverse 5'-TCCAGTTTGGTAGCATCCATC-3'

TGF $\beta$ (Tgfb 1) forward 5'-CCTGAGTGGCTGTCTTTTGAC-3'

TGF $\beta$ (Tgfb 1) reverse 5'-CCTGTATTCCGTCTCCTTGGT-3'

IL-17 (//17a) forward 5'-GGACTCTCCACCGCAATGA-3'

IL-17 (//17a) reverse 5'-GACCAGGATCTCTTGCTGGA-3'

TCR $\beta($ Tcrb-C) forward 5'-CTGCCAAGTGCAGTTCCAT-3'

TCR $\beta$ (Tcrb-C) reverse 5'-GGCCTCTGCACTGATGTTCT-3'

\section{Flow cytometry}

Splenocytes were labeled with FITC-conjugated anti-CD3, PE-conjugated anti-CD4 and CY5-conjugated anti-CD8 (PharMingen, San Jose, CA). Cell 

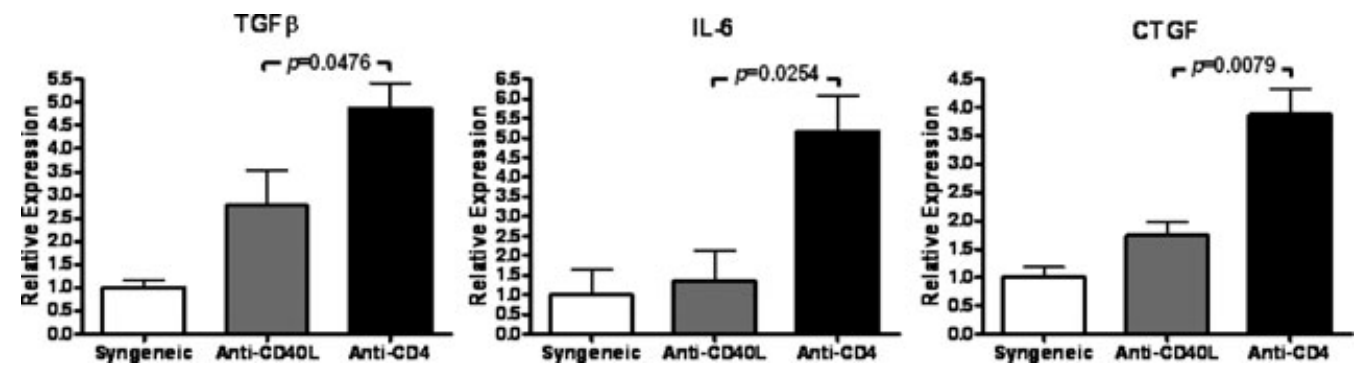

Figure 1: Elevated intragraft expression of TGF $\beta$, IL-6 and connective tissue growth factor (CTGF) in cardiac allografts undergoing chronic rejection (CR). TGF $\beta$, IL-6 and CTGF message levels were determined at day 30 posttransplant using quantitative real-time PCR in syngeneic cardiac grafts, cardiac allografts from recipients treated with anti-CD40L mAb therapy (Anti-CD40L) or cardiac allografts whose recipients were transiently depleted of CD4+ cells (Anti-CD4). Bars represent mean + S.E.M. of four to nine grafts with expression relative to GAPDH normalized to the syngeneic group.

analyses were performed on lymphocytes gated using forward vs. side scatter using a Becton Dickinson FACSCalibur (San Jose, CA).

\section{Statistical analysis}

Statistical significance was calculated using an unpaired $t$-test with Welch's correction. $p$-values $\leq 0.05$ were considered statistically significant.

\section{Results}

\section{Experimental system}

BALB/C cardiac allografts in C57BL/6 recipients receiving anti-CD40L mAb continue to function for $>60$ days and do not develop CR, unless transduced with TGF $\beta$ (8). In contrast, allografts in recipients transiently depleted of CD4+ cells develop CR as CD4+ cells begin to repopulate the periphery between 3 and 4 weeks following initial depletion (8,39-41). Echocardiographic and histologic analysis revealed that day 30 posttransplant represents a critical point in this CR model as extensive graft hypertrophy and fibrosis are present at this time and are followed by degradation of cardiac contractility (26). Therefore, grafts were assessed at day 30 posttransplant in these studies. We have used these models to better understand the roles of TGF $\beta$, IL-6 and CTGF in CR.

\section{Elevated intragraft TGFB, IL-6 and CTGF expression correlate with $\mathrm{CR}$}

Transduction of allografts, but not syngeneic grafts, with TGF $\beta$ is sufficient to induce CTGF and CR (8), indicating the involvement of an immune component in TGF $\beta$-mediated fibrosis. This is further supported by our recent identification of IL-6 as a critical inducer of CR (26). Hence, the in vivo interactions of TGF $\beta$, CTGF and IL- 6 in CR were the focus of this study. TGF $\beta$, CTGF and IL-6 transcripts were measured in grafts whose recipients were transiently depleted of CD4+ cells, which develop CR, and compared to allografts whose recipients were treated with anti-CD40L, which do not develop $\mathrm{CR}$, or untreated syngeneic grafts. Intragraft levels of TGF $\beta$, IL-6 and CTGF were significantly increased ( $p=0.0476,0.0254$ and 0.0079 , respectively) in cardiac allografts whose recipients were transiently de- pleted of CD4+ cells than in grafts whose recipients were treated with anti-CD40L or syngeneic controls (Figure 1). Thus, the upregulation of all three cytokines was observed in grafts undergoing $\mathrm{CR}$.

\section{Forced expression of CTGF or TGF promotes allograft fibrosis}

To determine whether exogenous expression of CTGF promotes cardiac fibrosis, allografts and syngeneic grafts were transduced with AdCTGF. AdCTGF transduction of allografts in recipients treated with anti-CD40L caused a significant increase in fibrotic area by day 30 posttransplant compared to allografts transduced with control virus (Figure 2A). In contrast, syngeneic grafts transduced with AdCTGF had similar levels of fibrosis to controls. It should be noted that the mean fibrotic area for AdCTGFtransduced allografts was less than in hearts transduced with AdTGF $\beta$, consistent with previous descriptions in lung transductions (42). This difference could not be accounted for by differences in transgene expression levels, as AdTGF $\beta$ and AdCTGF expression were comparable in these studies as determined by real-time PCR (data not shown). Thus, while forced expression of either TGF $\beta$ or CTGF promoted cardiac allograft fibrosis, they did so to different extents (Figure 2). This could in part be due to TGF $\beta$ induction of endogenous CTGF expression $(8,17,43)$, thereby producing an additive effect.

It has been observed that TGF $\beta$ and CTGF are potently fibrotic in tandem while less fibrotic individually $(44,45)$. Therefore, we asked whether cotransduction of both TGF $\beta$ and CTGF vectors would induce fibrosis and CR in syngeneic grafts. No increases in fibrosis were observed upon cotransduction of syngeneic grafts compared to single virus transduction (data not shown). Thus, while injection of TGF $\beta$ and CTGF synergize to cause fibrotic responses in the skin (45), forced expression of both was insufficient to induce fibrosis or CR in syngeneic cardiac grafts, further supporting the requirement of an immune component. 

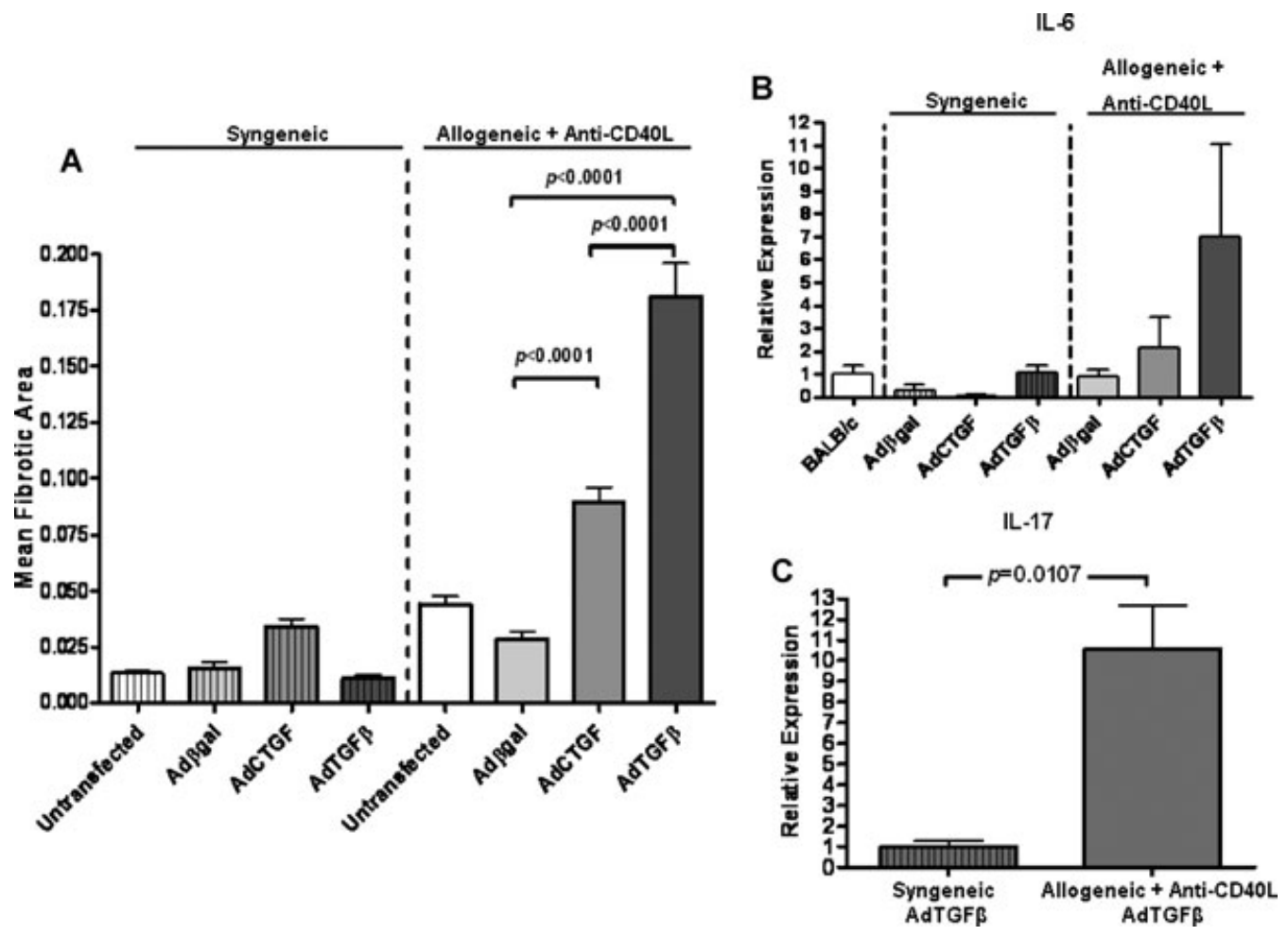

Figure 2: Forced expression of TGF $\beta$ or connective tissue growth factor (CTGF) promotes allograft fibrosis. (A) Morphometric analysis of Masson's trichrome staining at day 30 posttransplant in cardiac grafts that were left untransduced or transduced with adenoviral vectors encoding $\beta$ gal (Ad $\beta$ gal), CTGF (AdCTGF) or TGF $\beta$ (AdTGF $\beta$ ) before grafting into syngeneic recipients or allogeneic recipients treated with anti-CD40L. Bars represent the combined mean + S.E.M. of fibrotic (blue) area of 10-12 frames of view per heart taken from 5 to 12 different cardiac grafts per group. (B) Intragraft IL-6 message levels were determined at day 30 posttransplant using quantitative real-time PCR in groups from (A). Bars represent mean + S.E.M. of at least four hearts per group with expression relative to GAPDH normalized to naïve, untransplanted BALB/c hearts. (C) Intragraft IL-17 message levels were determined using quantitative real-time PCR in syngeneic grafts transduced with AdTGF $\beta$ or allogeneic grafts transduced with AdTGF $\beta$ whose recipients received anti-CD40L treatment. Bars represent mean + S.E.M. of at least five independent hearts per group with expression relative to GAPDH normalized to the naïve BALB/c group.

We next considered whether the greater fibrotic activity of AdTGF $\beta$ relative to AdCTGF could be due to immunologic effects. TGF $\beta$ is chemotactic for multiple immune cell types (10) that are able to produce IL-6, which we have recently reported to play a critical role in CR (26). Therefore, we asked whether differences in intragraft IL-6 expression might account for these disparate outcomes. IL-6 transcript levels exhibited a suggested increase in AdTGF $\beta$, but not AdCTGF transduced allografts whose recipients received anti-CD40L therapy. No increases in IL-6 expression were observed in AdTGF $\beta$ or AdCTGF-transduced syngeneic grafts (Figure 2B).

TGF $\beta$ and IL-6 have been implicated in the development of Th17 responses (27), which have recently been linked to CR $(46,47)$. Hence, we assessed the expression of IL-17 in allogeneic and syngeneic grafts transduced with AdTGF $\beta$. IL-17 expression was significantly greater $(p=0.0107)$ in allografts than in syngeneic grafts (Figure 2C), whereas IL-17 expression was similar in allogeneic and syngeneic grafts transduced with AdCTGF (data not shown). Thus, increased IL-17 and CTGF transcript levels may promote fibrosis associated with AdTGF $\beta$-transduced allografts, but not AdTGF $\beta$-transduced syngeneic grafts that do not develop fibrosis.

\section{IL-6 neutralization reduces intragraft CTGF and IL-17 transcripts}

The association between TGF $\beta$, IL-6 and CTGF (Figure 1) may be strengthened by previous reports that IL-6 enhances TGF $\beta$ signaling by altering receptor localization in the cell membrane (29) and that IL-6 can alter the outcome of TGF $\beta$ signaling $(27,28)$. Indeed, we have previously reported that IL-6 neutralization prevents CR of cardiac allografts (26). We therefore asked whether IL-6 neutralization would inhibit CTGF or IL-17 expression (Figure 3). In allografts whose recipients were transiently depleted of CD4+ cells, treatment with anti-IL-6 mAb significantly reduced intragraft IL-6, IL-17 and CTGF expression ( $p=0.0216,0.0044$ and 0.0180 , respectively) compared to control antibody treatment. In contrast, TGF $\beta$ expression levels remained 

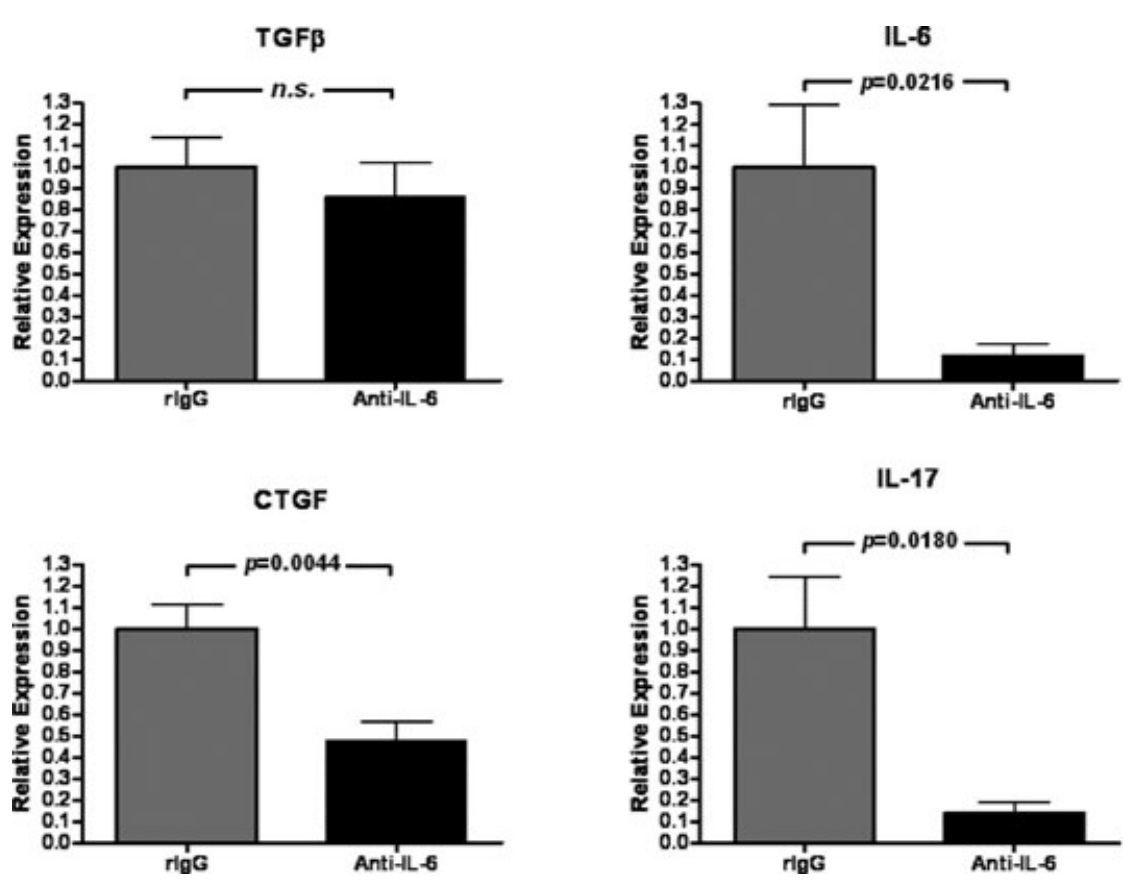

Figure 3: IL-6 neutralization reduces expression of IL-6, IL-17 and connective tissue growth factor (CTGF) but not TGF $\beta$ in cardiac allografts undergoing chronic rejection (CR). Intragraft IL-6, IL-17, CTGF and TGF $\beta$ message levels were determined at day 30 posttransplant using quantitative realtime PCR in cardiac allograft recipients that were transiently depleted of CD4+ cells and received either neutralizing anti-IL-6 (Anti-IL-6) or control rat IgG (rlgG). Bars represent mean + S.E.M. of six to eight grafts per group with expression relative to GAPDH normalized against rlgG-treated controls. unchanged (Figure 3). Thus, IL-6 promotes intragraft IL-6, IL-17 and CTGF expression.

\section{CTGF neutralization ameliorates allograft fibrosis}

We next asked whether CTGF neutralization would inhibit the fibrosis associated with CR. To this end, we treated allograft recipients that were transiently depleted of CD4+ cells with neutralizing anti-CTGF mAb or control antibody. Treatment with anti-CTGF $m A b$ resulted in significant reduction of fibrotic area ( $p<0.0001$, Figure $4 A, B)$, but was not accompanied by reduction of intragraft TGF $\beta$, CTGF or IL-6 transcripts (Figure 4C). These observations support a role for CTGF as a downstream mediator of fibrosis associated with $\mathrm{CR}$.

\section{CTGF neutralization decreases cardiomyocyte hypertrophy associated with $C R$}

CTGF can induce cardiomyocyte hypertrophy $(48,49)$, a function it shares with IL-6 (26). Because IL-6 neutralization inhibited CTGF expression (Figure 3), we assessed the effect of neutralizing CTGF on cardiomyocyte hypertrophy. Anti-CTGF treatment resulted in a significant decrease $(p<0.0001)$ in cardiomyocyte hypertrophy (Figure 5A) and significantly reduced $(p=0.0102)$ the intragraft expression of ANP (Figure 5B), a molecular marker of cardiac hypertrophy $(50,51)$. For reference, cardiomyocyte area and ANP expression levels for naïve, untransplanted BALB/c hearts and allografts transplanted into recipients receiving antiCD40L therapy are depicted.

\section{CTGF neutralization inhibits T-cell infiltration of grafts}

CTGF promotes integrin-mediated adhesive responses in multiple cell types (52-61) and induces the production of chemokines (62). We therefore asked whether CTGF neutralization might also alter the infiltration of immune cells into grafts undergoing CR. Histologic analysis indicated reduced cellular infiltrate in grafts receiving anti-CTGF (Figure 6A). Indeed, a significant decrease $(p=0.0238$ ) in TCR $\beta$ constant region expression, a marker of graftinfiltrating $T$ cells (63), was observed (Figure 6B). To verify that this difference was not due to CTGF neutralization preventing peripheral repopulation of CD4+ cells, we compared the percentage of CD4+ cells in anti-CTGF and control treated graft recipients. No significant differences were observed between these groups (Figure 6C).

\section{Discussion}

$\mathrm{CR}$ has been associated with multiple factors, perhaps most frequently with TGF $\beta$ (9). However, the role of TGF $\beta$ in $\mathrm{CR}$ is complicated by its pleiotropic activity encompassing immunosuppressive and antiproliferative effects in immune $(10,64-66)$ and nonimmune $(15,67)$ cells as well as the induction of Treg (68-70), which are associated with graft acceptance $(12,13,24)$. Thus, TGF $\beta$ may promote graft survival and global immune tolerance while suppressing malignancy, making it ill-suited as a therapeutic target in the treatment of $\mathrm{CR}$. This has prompted investigation into the downstream mediators of fibrotic TGF $\beta$ function $(8,23)$.

Multiple reports indicate that TGF $\beta$ requires additional factors to drive fibrosis $(8,44,45)$. Indeed, syngeneic grafts do not develop fibrosis in response to TGF $\beta$, while allografts whose recipients receive anti-CD40L mAb develop marked fibrosis in response to TGF $\beta$ (Figure 2; (8)). Hence, alloimmune responses potentiate the profibrotic effects of TGF $\beta$. 
A

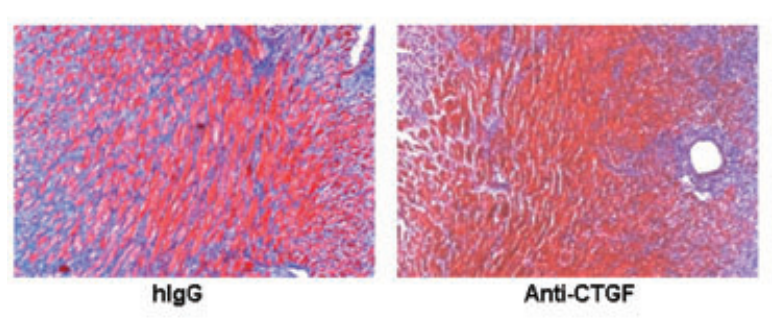

B

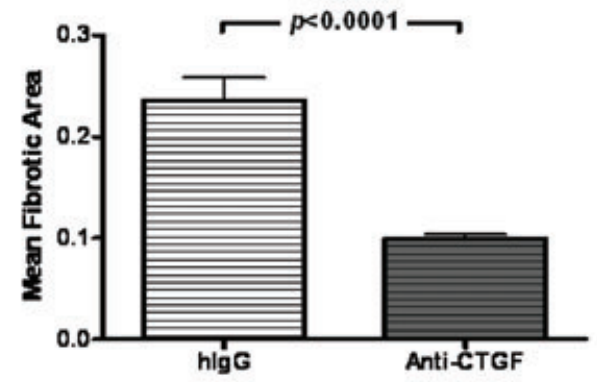

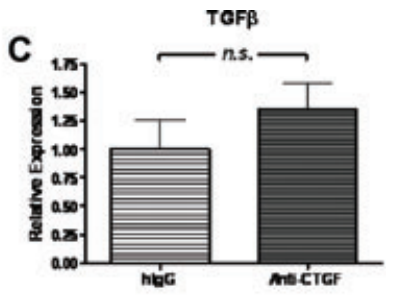
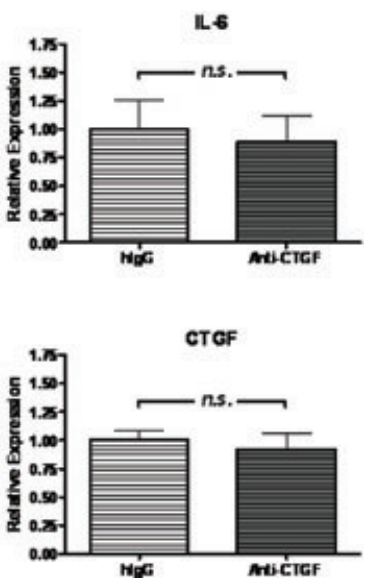

Figure 4: Connective tissue growth factor (CTGF) neutralization ameliorates fibrosis. (A) Representative sections of Masson's trichrome stains, in which fibrotic tissue stains blue, of cardiac allografts from recipients transiently depleted of CD4+ cells (Anti-CD4) at day 30 posttransplant in recipients treated with control lgG or neutralizing anti-CTGF mAb (200x magnification). (B) Morphometric analysis of trichrome staining of groups in (A). Bars represent mean + S.E.M. of 10-12 frames of view from each of six to nine hearts. (C) TGF $\beta$, IL-6 and CTGF message levels were determined at day 30 posttransplant using quantitative real-time PCR in cardiac allografts described in (A). Bars represent mean + S.E.M. of samples taken from 8 to 12 different cardiac grafts with expression relative to GAPDH normalized against hlgG-treated controls.

We have reported a critical role for IL-6 in CR (26), whose elevated expression correlated with TGF $\beta$ and CTGF (Figure 1). Correlations of TGF $\beta$ with CTGF (8) and IL-6 (26) have previously been described. Further, we have previously observed CTGF expression associated with areas of graft-infiltrating mononuclear cells (8), whose recruitment during inflammatory responses has been linked to IL-6 $(71,72)$. Therefore, we considered that there may be connectivity between all three cytokines.

To ascertain the sufficiency of TGF $\beta$ and CTGF to induce $\mathrm{CR}$, allogeneic and syngeneic cardiac grafts were transduced with AdTGF $\beta$ or AdCTGF and transplanted into recipients receiving anti-CD40L $\mathrm{mAb}$ or syngeneic recipients. AdTGF $\beta$ and AdCTGF significantly increased mean fibrotic area compared to untransduced or control vectortreated allografts (Figure 2A). Consistent with a previous report of adenoviral transduction of lungs (42), the fibrotic response to TGF $\beta$ transduction in the heart was significantly greater than the response to CTGF transduction (Figure 2A). Greater fibrotic responses to AdTGF $\beta$ could be from synergy of TGF $\beta$-induced immune factors and CTGF in cardiac allografts, an effect which is not observed in syngeneic grafts (8). Further, in cardiac allografts, TGF $\beta$ induction of endogenous CTGF may synergize with TGF $\beta$ - mediated chemotactic effects on multiple immune lineage cells (10), which may explain the suggested upregulation of IL-6 and significant upregulation of IL-17 (Figure 2).

Given the differences in AdTGF $\beta$ responses between allografts and syngeneic grafts and the correlation of TGF $\beta$ and CTGF with IL-6 in CR (Figure 1), we asked whether the presence of IL-6 was required for CTGF upregulation. In cardiac allograft recipients transiently depleted of CD4+ cells, IL-6 neutralization reduced the expression of IL-6 and CTGF without altering TGF $\beta$ transcript levels (Figure 3). This suggests that TGF $\beta$ transcript regulation lies upstream of IL-6 and CTGF in CR. It should be noted that IL-6 neutralization does not prevent repopulation of CD4+ cells in the periphery (26). This indicates that the ability of IL-6 neutralization to prevent $\mathrm{CR}$ (26) could function in part through reduction of intragraft CTGF. Further, IL-6 neutralization significantly inhibited IL-17 expression (Figure 3), indicating that IL-17 might play a role in CTGF induction, as IL-17 has been reported to induce collagen production in cardiac fibroblasts (73). Another explanation for this effect might be decreased recruitment of graft-infiltrating cells which may express or induce local cells to express CTGF (8), IL-6 and IL-17. Indeed, IL-6 induces chemotaxis and migration of immune cells $(74,75)$. 


\section{Booth et al.}

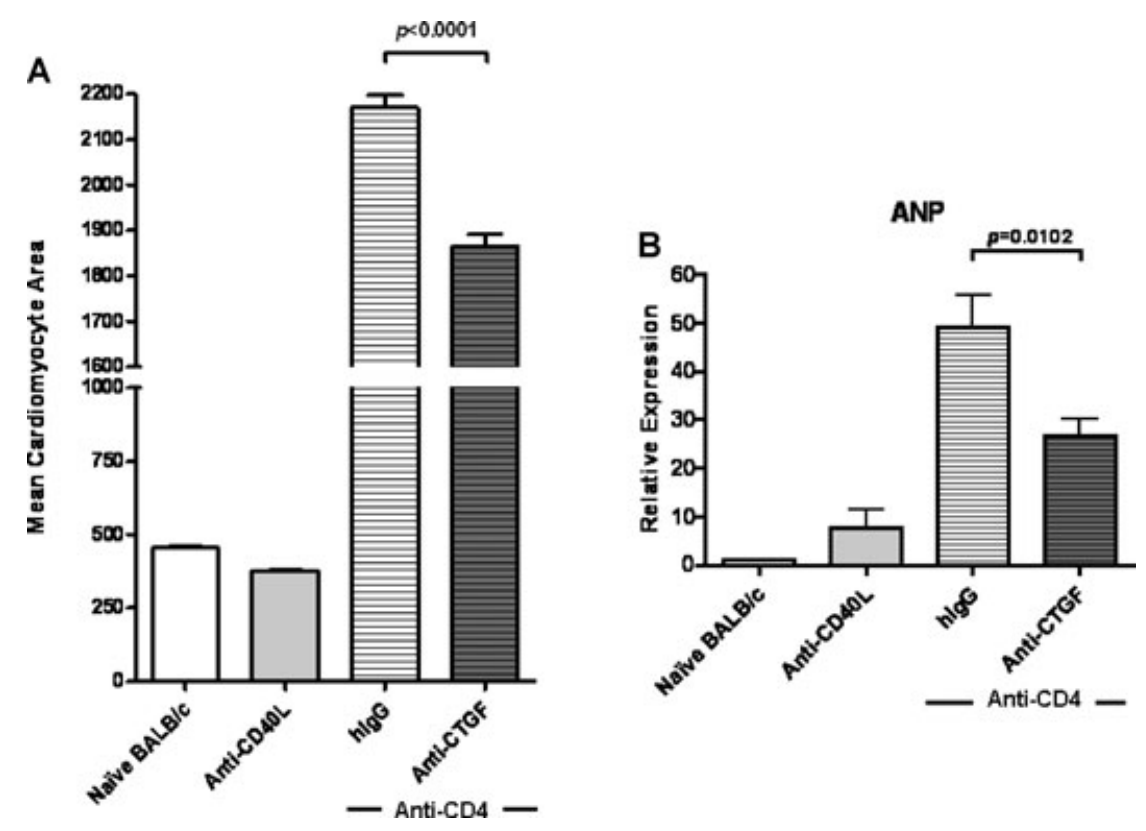

Figure 5: Connective tissue growth factor (CTGF) neutralization ameliorates cardiac hypertrophy in chronic rejection (CR) grafts. (A) Cardiomyocyte area was quantified from H\&E stains of day 30 posttransplant cardiac allografts taken from recipients transiently depleted of CD4+ cells (Anti-CD4) and receiving CTGF-neutralizing mAb (Anti-CTGF) or control antibodies (hlgG), recipients treated with Anti-CD40L mAb, or naïve, untransplanted BALB/c hearts. Bars represent mean + S.E.M. of area measurements taken from $\geq 100$ cardiomyocytes per heart from 5 (naïve BALB/c and Anti-CD40L), 8 (Anti-CD4 + hlgG) or 10 (Anti-CD4 + Anti-CTGF) different hearts per group. (B) Intragraft message levels of atrial natriuretic peptide (ANP), a marker of cardiac hypertrophy, were quantified with real-time PCR in cardiac grafts from groups in (A) at day 30 posttransplant. Bars represent mean + S.E.M. of 8-12 grafts per experimental group (Anti-CD4 + hlgG or Anti-CTGF) and four grafts per control group (Anti-CD40L and naïve BALB/c) with expression relative to GAPDH normalized against the naïve BALB/c hearts.

As IL-6 neutralization ameliorated CR (26) and decreased intragraft CTGF expression (Figure 3), we treated cardiac allograft recipients with neutralizing CTGF mAb. CTGF neutralization significantly reduced allograft fibrosis (Figure 4A, B) without significantly reducing intragraft TGF $\beta$, IL-6 or CTGF expression (Figure 4C). These findings are consistent with CTGF being a downstream mediator of fibrosis in $\operatorname{CR}(16,23,42,76)$.

The significant but incomplete reduction in fibrotic area in response to CTGF neutralization may be explained by multiple factors. Our neutralization protocol, though effective, may not be optimal. Another possibility is the presence of CTGF-independent profibrotic effects of TGF $\beta$ and/or IL-6 (77). A further consideration is whether the mAb FG-3019, which recognizes CTGF module 2 in humans and rodents (33), might inhibit some but not all profibrotic effects of CTGF. However, this possibility seems unlikely in light of a recent report evaluating the antifibrotic efficacy of antiCTGF antibodies directed against each of the four CTGF modules. In this report, only mAb directed against the von Willebrand factor type $\mathrm{C}$ domain (module 2 ) was able to inhibit TGF $\beta$-induced fibrosis (78). Indeed, this is the same domain that the anti-CTGF mAb utilized in our study binds $(32,33)$
Beyond its roles in fibrosis, CTGF can exert other effects relevant to CR. Recent studies have described a concomitance of cardiomyocyte hypertrophy with CR $(26,79,80)$. CTGF is produced by hypertrophic chondrocytes during development (81), and is produced by cardiac myocytes in response to hypertrophic stimuli (49). In addition, CTGF itself can induce cardiomyocyte hypertrophy (48). Treatment with neutralizing anti-CTGF mAb significantly reduced mean cardiomyocyte area (Figure 5A) and intragraft levels of ANP (Figure 5B), a marker of cardiac hypertrophy in multiple settings $(26,50,51)$. However, it should be noted that anti-CTGF mAb did not inhibit cardiac hypertrophy to the extent previously observed with anti-IL-6 (26). This finding indicates that in addition to driving cardiac fibrosis, CTGF may augment cardiomyocyte hypertrophy associated with CR. Interestingly, hypertrophy is associated with downregulation of two recently discovered CTGF-inhibiting micro RNAs in cardiac myocytes (82). Thus, CTGF may be linked to cardiac hypertrophy on multiple levels.

Finally, as CTGF is known to play an important role in fibroblast adhesion in response to TGF $\beta(55,76)$, we asked whether CTGF might similarly influence recruitment of lymphocytes to the graft. Histologic assessment of infiltrating cells was indicative of reduced numbers of 
A

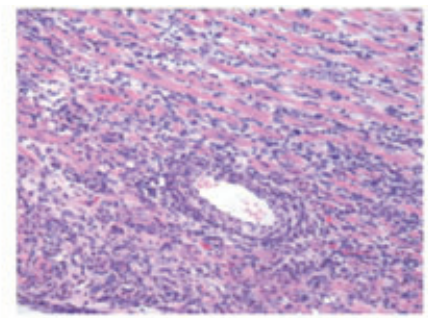

hlgG

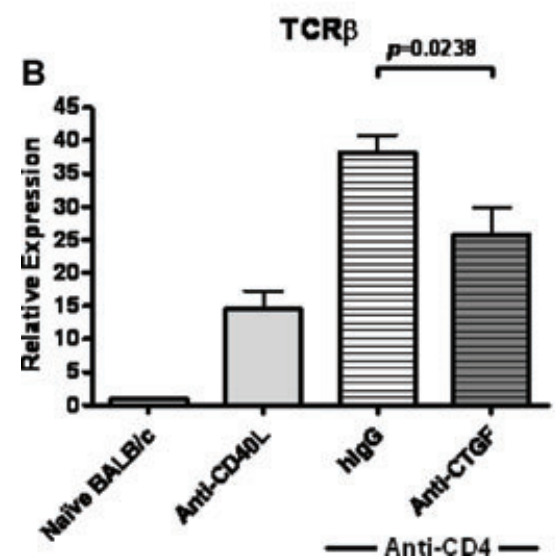

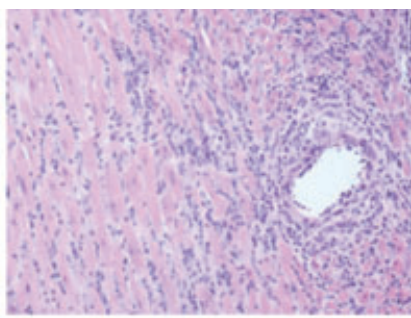

Anti-CTGF

C

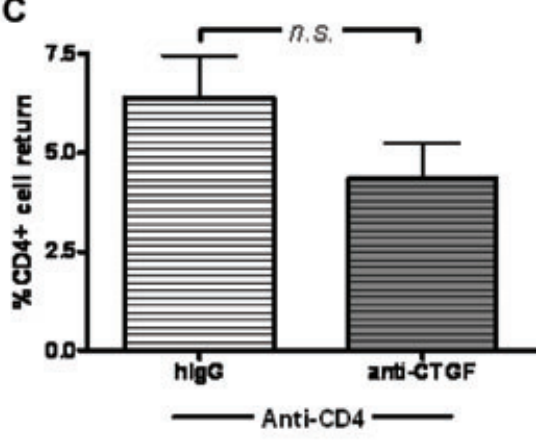

Figure 6: Connective tissue growth factor (CTGF) neutralization limits graft infiltration by T cells in chronic rejection (CR) grafts. (A) Representative H\&E stains of day 30 posttransplant cardiac allografts taken from recipients transiently depleted of CD4+ cells (AntiCD4) and receiving CTGF neutralizing mAb (Anti-CTGF) or control antibodies (hlgG). Stains suggest a reduction in perivascular infiltrate density in grafts treated with neutralizing Anti-CTGF. (B) Intragraft message levels of T-cell receptor $\beta$ constant region (TCR $\beta$ ) were quantified at day 30 posttransplant with real-time PCR as a measure of T-cell infiltration of allografts in recipients transiently depleted of CD4+ cells (Anti-CD4) and receiving anti-CTGF mAb or control hlgG antibodies, recipients treated with Anti-CD40L mAb, or naïve BALB/c hearts. Bars represent mean + S.E.M. of 8-12 grafts per group with expression relative to GAPDH normalized against the hlgG group. (C) Repopulation of CD4+ cells in the periphery at day 30 posttransplant was determined by flow cytometric analysis of splenocytes isolated from graft recipients. Bars represent mean + S.E.M. of the percentage CD4+ cells of the gated cell population in five to seven recipients tested.

graft-infiltrating lymphocytes (Figure 6A). This observation was further supported by significant reduction of intragraft TCR $\beta$ constant region expression (Figure 6B) in response to CTGF neutralization.

\section{A}

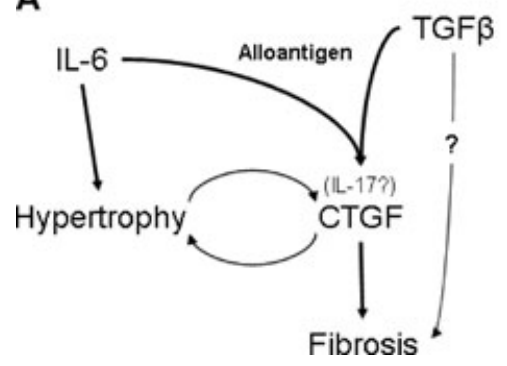

On the basis of these observations and others in the literature, we propose a model representing the interactions of TGF $\beta$, IL-6 and CTGF and their induction of hypertrophy and fibrosis associated with $\mathrm{CR}$

B

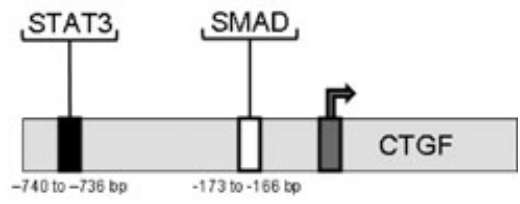

Figure 7: Proposed model of cytokine interactions in chronic rejection. (A) In cardiac allografts, TGF $\beta$ and IL-6 contribute to CTGF production. IL-6 and CTGF are both known to promote hypertrophy in cardiac myocytes, which in turn can produce CTGF. CTGF functions as a downstream mediator of fibrosis. (B) Induction of CTGF downstream of TGF $\beta$ and IL-6 could be explained by the respective presence of a consensus SMAD-binding element and a STAT3 response element in 5' region upstream of the CTGF promoter. For expanded explanations, please see text. 
(Figure 7A). In cardiac allografts that undergo CR, TGF $\beta$ (Figure 1 (8)) and IL-6 (Figures 1 and 2B (26)) are induced. In syngeneic grafts, forced expression of TGF $\beta$ is insufficient to upregulate CTGF and fibrosis (8), and IL-6 remains at basal levels (Figure 2B). IL-6 neutralization inhibits hypertrophy and fibrosis associated with CR (26), which may be in part through inhibition of CTGF and IL-17 expression whereas TGF $\beta$ expression remains unchanged (Figure 3). Thus, TGF $\beta$ and IL-6 appear to be cooperative upstream factors promoting CTGF expression and CR. CTGF neutralization limits fibrosis (Figure 4A, B) and cardiomyocyte hypertrophy (Figure 5), without altering intragraft TGF $\beta$, IL6 or CTGF transcripts (Figure $4 \mathrm{C}$ ). These effects of CTGF neutralization coincide with reduction in graft-infiltrating $T$ cells (Figure 6). Together, these observations support a downstream role for CTGF in fibrosis and hypertrophy.

Contexts in which TGF $\beta$ and IL-6 are present coincide with intragraft IL-17 expression, which has been implicated in promoting cardiac remodeling (83), fibrosis (73), bronchiolitis obliterans syndrome in lung transplant patients (46) and cardiac allograft vasculopathy (47). However, the effects of IL-17 on hypertrophy and CTGF expression are unclear and merit further investigation. Our proposed model of CTGF induction downstream of IL-6 and TGF $\beta$ (and perhaps IL17) might be explained by previous identification of both a STAT3 responsive element $(-740$ to $-736 \mathrm{bp})(84)$ and a consensus SMAD-binding element (-173 and -166) (85) upstream of the CTGF promoter (Figure 7B). Hence, optimal induction of CTGF in CR may require that CTGF producing cells receive both SMAD and STAT3 signals, likely provided by TGF $\beta$ (10) and IL-6 (86), respectively.

This study supports a role for CTGF as a downstream mediator of fibrosis and highlights the essential contributions of immune elements to $\mathrm{CR}$ and fibrosis of cardiac grafts while elucidating relationships between TGF $\beta$, IL-6 and CTGF. Further, these studies indicate for the first time that $m A b$ neutralizing CTGF can ameliorate fibrosis and hypertrophy associated with CR. These findings further implicate IL-6 as a critical immune factor in CR that may potentiate TGF $\beta$ mediated CTGF induction. Finally, TGF $\beta$-mediated induction of fibrosis in allogeneic but not syngeneic grafts was associated with a suggested increase in intragraft IL-6 expression and a significant increase in IL-17 expression, supporting the notion that TGF $\beta$ induction of fibrosis and CR requires interaction with immune parameters.

\section{Acknowledgments}

Supported by an NIH grants R01 HL070613 (DKB) and R01 Al061469 (DKB) and by an American Heart Association Predoctoral Fellowship (AJB).

\section{References}

1. Orosz CG, Pelletier RP. Chronic remodeling pathology in grafts. Curr Opin Immunol 1997; 9: 676-680.
2. Paul LC. Current knowledge of the pathogenesis of chronic allograft dysfunction. Transplant Proc 1999; 31: 1793-1795.

3. Waaga AM, Gasser M, Laskowski I, Tilney NL. Mechanisms of chronic rejection. Curr Opin Immunol 2000; 12: 517-521.

4. Womer KL, Vella JP, Sayegh MH. Chronic allograft dysfunction: Mechanisms and new approaches to therapy. Semin Nephrol 2000; 20: 126-147.

5. Weiss MJ, Madsen JC, Rosengard BR, Allan JS. Mechanisms of chronic rejection in cardiothoracic transplantation. Front Biosci 2008; 13: 2980-2988.

6. Mehra MR. Contemporary concepts in prevention and treatment of cardiac allograft vasculopathy. Am J Transplant 2006; 6: 12481256.

7. Valantine H. Cardiac allograft vasculopathy after heart transplantation: Risk factors and management. J Heart Lung Transplant 2004; 23(5 Suppl): S187-S193.

8. Csencsits K, Wood SC, Lu G et al. Transforming growth factor beta-induced connective tissue growth factor and chronic allograft rejection. Am J Transplant 2006; 6(5 Pt 1): 959-966.

9. Jain S, Furness PN, Nicholson ML. The role of transforming growth factor beta in chronic renal allograft nephropathy. Transplantation 2000; 69: 1759-1766.

10. Li MO, Wan YY, Sanjabi S, Robertson AK, Flavell RA. Transforming growth factor-beta regulation of immune responses. Annu Rev Immunol 2006; 24: 99-146.

11. Blobe GC, Schiemann WP, Lodish HF. Role of transforming growth factor beta in human disease. N Engl J Med 2000; 342: 1350-1358.

12. Wood KJ, Sakaguchi S. Regulatory T cells in transplantation tolerance. Nat Rev Immunol 2003; 3: 199-210.

13. Yong $Z$, Chang $L, M e i ~ Y X, Y i L$. Role and mechanisms of $\mathrm{CD} 4+\mathrm{CD} 25+$ regulatory $T$ cells in the induction and maintenance of transplantation tolerance. Transpl Immunol 2007; 17: 120-129.

14. Walsh PT, Taylor DK, Turka LA. Tregs and transplantation tolerance. J Clin Invest 2004; 114: 1398-1403.

15. Brattain MG, Markowitz SD, Willson JK. The type II transforming growth factor-beta receptor as a tumor-suppressor gene. Curr Opin Oncol 1996; 8: 49-53.

16. Leask A, Abraham DJ. The role of connective tissue growth factor, a multifunctional matricellular protein, in fibroblast biology. Biochem Cell Biol 2003; 81: 355-363.

17. Chen MM, Lam A, Abraham JA, Schreiner GF, Joly AH. CTGF expression is induced by TGF-beta in cardiac fibroblasts and cardiac myocytes: A potential role in heart fibrosis. J Mol Cell Cardiol 2000; 32: 1805-1819.

18. de Winter P, Leoni $P$, Abraham D. Connective tissue growth factor: Structure-function relationships of a mosaic, multifunctional protein. Growth Factors 2008; 26: 80-91.

19. Bonniaud P, Martin G, Margetts PJ et al. Connective tissue growth factor is crucial to inducing a profibrotic environment in "fibrosisresistant" BALB/c mouse lungs. Am J Respir Cell Mol Biol 2004; 31: 510-516.

20. Cheng O, Thuillier R, Sampson E et al. Connective tissue growth factor is a biomarker and mediator of kidney allograft fibrosis. Am J Transplant 2006; 6: 2292-2306.

21. Yuan YC, Xia ZK, Mu JJ, Zhang QC, Yin BL. Increased connective tissue growth factor expression in a rat model of chronic heart allograft rejection. J Formos Med Assoc 2009; 108: 240-246.

22. Daniels A, van Bilsen M, Goldschmeding R, Van Der Vusse GJ, van Nieuwenhoven FA. Connective tissue growth factor and cardiac fibrosis. Acta Physiol (Oxf) 2009; 195: 321-338.

23. Mannon RB. Therapeutic targets in the treatment of allograft fibrosis. Am J Transplant 2006; 6(5 Pt 1): 867-875.

24. Csencsits K, Wood SC, Lu G, Bishop DK. Transforming growth factor-beta1 gene transfer is associated with the 


\section{TGF $\beta$ and IL-6 Promote CTGF and Fibrosis}

development of regulatory cells. Am J Transplant 2005; 5: 23782384.

25. Guo X, Wang XF. Signaling cross-talk between TGF-beta/BMP and other pathways. Cell Res 2009; 19: 71-88.

26. Diaz JA, Booth AJ, Lu G, Wood SC, Pinsky DJ, Bishop DK. Critical role for IL-6 in hypertrophy and fibrosis in chronic cardiac allograft rejection. Am J Transplant 2009; 9: 1773-1783.

27. Bettelli E, Carrier Y, Gao W et al. Reciprocal developmental pathways for the generation of pathogenic effector $\mathrm{TH} 17$ and regulatory T cells. Nature 2006; 441: 235-238.

28. Chen RH, Chang MC, Su YH, Tsai YT, Kuo ML. Interleukin-6 inhibits transforming growth factor-beta-induced apoptosis through the phosphatidylinositol 3-kinase/Akt and signal transducers and activators of transcription 3 pathways. J Biol Chem 1999; 274: 23013-23019.

29. Zhang $X L$, Topley $N$, Ito $T$, Phillips A. Interleukin-6 regulation of transforming growth factor (TGF)-beta receptor compartmentalization and turnover enhances TGF-beta1 signaling. J Biol Chem 2005; 280: 12239-12245.

30. Corry RJ, Winn HJ, Russell PS. Primarily vascularized allografts of hearts in mice. The role of $\mathrm{H}-2 \mathrm{D}, \mathrm{H}-2 \mathrm{~K}$, and non- $\mathrm{H}-2$ antigens in rejection. Transplantation 1973; 16: 343-350.

31. Burrell BE, Csencsits K, Lu G, Grabauskiene S, Bishop DK. CD8+ Th17 mediate costimulation blockade-resistant allograft rejection in T-bet-deficient mice. J Immunol 2008; 181: 3906-3914.

32. Aikawa T, Gunn J, Spong SM, Klaus SJ, Korc M. Connective tissue growth factor-specific antibody attenuates tumor growth, metastasis, and angiogenesis in an orthotopic mouse model of pancreatic cancer. Mol Cancer Ther 2006; 5: 1108-1116.

33. Dornhofer N, Spong S, Bennewith K et al. Connective tissue growth factor-specific monoclonal antibody therapy inhibits pancreatic tumor growth and metastasis. Cancer Res 2006; 66: 58165827.

34. Chan SY, Goodman RE, Szmuszkovicz JR, Roessler B, Eichwald EJ, Bishop DK. DNA-liposome versus adenoviral mediated gene transfer of transforming growth factor beta1 in vascularized cardiac allografts: Differential sensitivity of CD4+ and CD8+ T cells to transforming growth factor beta1. Transplantation 2000; 70: 1292 1301.

35. Chan SY, Li K, Piccotti JR et al. Tissue-specific consequences of the anti-adenoviral immune response: Implications for cardiac transplants. Nat Med 1999; 5: 1143-1149.

36. Haberberger TC, Kupfer K, Murphy JE. Profiling of genes which are differentially expressed in mouse liver in response to adenoviral vectors and delivered genes. Gene Ther 2000; 7: 903-909.

37. Burrell BE, Lu G, Li XC, Bishop DK. OX40 costimulation prevents allograft acceptance induced by CD40-CD40L blockade. J Immunol 2009; 182: 379-390.

38. Nozato $\mathrm{T}$, Ito $\mathrm{H}$, Tamamori $\mathrm{M}$ et al. $\mathrm{G} 1$ cyclins are involved in the mechanism of cardiac myocyte hypertrophy induced by angiotensin II. Jpn Circ J 2000; 64: 595-601.

39. Bishop DK, Li W, Chan SY, Ensley RD, Shelby J, Eichwald EJ. Helper T lymphocyte unresponsiveness to cardiac allografts following transient depletion of CD4-positive cells. Implications for cellular and humoral responses. Transplantation 1994; 58: 576584

40. Piccotti JR, Li K, Chan SY, Eichwald EJ, Bishop DK. Cytokine regulation of chronic cardiac allograft rejection: Evidence against a role for Th1 in the disease process. Transplantation 1999; 67: 15481555.

41. Csencsits K, Burrell BE, Lu G, Eichwald EJ, Stahl GL, Bishop DK. The classical complement pathway in transplantation: Unanticipated protective effects of $\mathrm{Clq}$ and role in in- ductive antibody therapy. Am J Transplant 2008; 8: 16221630.

42. Bonniaud P, Margetts PJ, Kolb M et al. Adenoviral gene transfer of connective tissue growth factor in the lung induces transient fibrosis. Am J Respir Crit Care Med 2003; 168: 770-778.

43. Grotendorst GR, Okochi H, Hayashi N. A novel transforming growth factor beta response element controls the expression of the connective tissue growth factor gene. Cell Growth Differ 1996: 7: 469-480

44. Frazier K, Williams S, Kothapalli D, Klapper H, Grotendorst GR. Stimulation of fibroblast cell growth, matrix production, and granulation tissue formation by connective tissue growth factor. J Invest Dermatol 1996; 107: 404-411.

45. Mori T, Kawara S, Shinozaki M et al. Role and interaction of connective tissue growth factor with transforming growth factor-beta in persistent fibrosis: A mouse fibrosis model. J Cell Physiol 1999; 181: 153-159.

46. Burlingham WJ, Love RB, Jankowska-Gan E et al. IL-17-dependent cellular immunity to collagen type $\mathrm{V}$ predisposes to obliterative bronchiolitis in human lung transplants. J Clin Invest 2007; 117: 3498-3506.

47. Yuan X, Paez-Cortez J, Schmitt-Knosalla I et al. A novel role of CD4 Th17 cells in mediating cardiac allograft rejection and vasculopathy. J Exp Med 2008; 205: 3133-3144.

48. Hayata N, Fujio $Y$, Yamamoto $Y$ et al. Connective tissue growth factor induces cardiac hypertrophy through Akt signaling. Biochem Biophys Res Commun 2008; 370: 274-278.

49. Matsui Y, Sadoshima J. Rapid upregulation of CTGF in cardiac myocytes by hypertrophic stimuli: Implication for cardiac fibrosis and hypertrophy. J Mol Cell Cardiol 2004; 37: 477-481.

50. Caron KM, James LR, Kim HS et al. Cardiac hypertrophy and sudden death in mice with a genetically clamped renin transgene. Proc Natl Acad Sci USA 2004; 101: 3106-3111.

51. Fredj S, Bescond J, Louault C, Potreau D. Interactions between cardiac cells enhance cardiomyocyte hypertrophy and increase fibroblast proliferation. J Cell Physiol 2005; 202: 891-899.

52. Babic AM, Chen CC, Lau LF. Fisp12/mouse connective tissue growth factor mediates endothelial cell adhesion and migration through integrin alphavbeta3, promotes endothelial cell survival, and induces angiogenesis in vivo. Mol Cell Biol 1999; 19: 29582966.

53. Ball DK, Rachfal AW, Kemper SA, Brigstock DR. The heparinbinding $10 \mathrm{kDa}$ fragment of connective tissue growth factor (CTGF) containing module 4 alone stimulates cell adhesion. J Endocrinol 2003; 176: R1-R7.

54. Chen CC, Chen N, Lau LF. The angiogenic factors Cyr61 and connective tissue growth factor induce adhesive signaling in primary human skin fibroblasts. J Biol Chem 2001; 276: 10443-10452.

55. Chen $Y$, Abraham DJ, Shi-Wen $X$ et al. CCN2 (connective tissue growth factor) promotes fibroblast adhesion to fibronectin. Mol Biol Cell 2004; 15: 5635-5646.

56. Gao R, Brigstock DR. Connective tissue growth factor (CCN2) induces adhesion of rat activated hepatic stellate cells by binding of its C-terminal domain to integrin alpha(v)beta(3) and heparan sulfate proteoglycan. J Biol Chem 2004; 279: 8848-8855.

57. Hoshijima M, Hattori T, Inoue M et al. CT domain of CCN2/CTGF directly interacts with fibronectin and enhances cell adhesion of chondrocytes through integrin alpha5beta1. FEBS Lett 2006; 580: 1376-1382.

58. Nishida T, Kawaki H, Baxter RM, Deyoung RA, Takigawa M, Lyons KM. CCN2 (Connective Tissue Growth Factor) is essential for extracellular matrix production and integrin signaling in chondrocytes. J Cell Commun Signal 2007; 1: 45-58. 
59. Gao R, Brigstock DR. A novel integrin alpha5beta1 binding domain in module 4 of connective tissue growth factor (CCN2/CTGF) promotes adhesion and migration of activated pancreatic stellate cells. Gut 2006; 55: 856-862.

60. Heng EC, Huang Y, Black SA Jr, Trackman PC. CCN2, connective tissue growth factor, stimulates collagen deposition by gingival fibroblasts via module 3 and alpha6- and beta1 integrins. J Cell Biochem 2006; 98: 409-420.

61. Jedsadayanmata A, Chen CC, Kireeva ML, Lau LF, Lam SC. Activation-dependent adhesion of human platelets to Cyr61 and Fisp12/mouse connective tissue growth factor is mediated through integrin alpha(llb)beta(3). J Biol Chem 1999; 274: $24321-$ 24327.

62. Wu SH, Lu C, Dong L, Chen ZQ. Signal transduction involved in CTGF-induced production of chemokines in mesangial cells. Growth Factors 2008; 26: 192-200.

63. Zhao XM, Frist WH, Yeoh TK, Miller GG. Expression of cytokine genes in human cardiac allografts: Correlation of IL-6 and transforming growth factor-beta (TGF-beta) with histological rejection. Clin Exp Immunol 1993; 93: 448-451.

64. Letterio JJ, Roberts AB. Regulation of immune responses by TGFbeta. Annu Rev Immunol 1998; 16: 137-161.

65. Prud'homme GJ, Piccirillo CA. The inhibitory effects of transforming growth factor-beta-1 (TGF-beta1) in autoimmune diseases. J Autoimmun 2000; 14: 23-42.

66. Wahl SM. Transforming growth factor beta: The good, the bad, and the ugly. J Exp Med 1994; 180: 1587-1590.

67. Massague J, Blain SW, Lo RS. TGFbeta signaling in growth control, cancer, and heritable disorders. Cell 2000; 103: 295-309.

68. Fu S, Zhang N, Yopp AC et al. TGF-beta induces Foxp3 +Tregulatory cells from CD4+ CD25- precursors. Am J Transplant 2004; 4: 1614-1627.

69. Chen $W$, Jin $W$, Hardegen $N$ et al. Conversion of peripheral CD4+CD25- naive $T$ cells to $C D 4+C D 25+$ regulatory $T$ cells by TGF-beta induction of transcription factor Foxp3. J Exp Med 2003; 198: 1875-1886.

70. Zheng SG, Gray JD, Ohtsuka K, Yamagiwa S, Horwitz DA. Generation ex vivo of TGF-beta-producing regulatory $T$ cells from CD4+CD25- precursors. J Immunol 2002; 169: 4183-4189.

71. Marin V, Montero-Julian FA, Gres S et al. The IL-6-soluble IL6Ralpha autocrine loop of endothelial activation as an intermediate between acute and chronic inflammation: An experimental model involving thrombin. J Immunol 2001; 167: 3435-3442.

72. Hurst SM, Wilkinson TS, McLoughlin RM et al. II-6 and its soluble receptor orchestrate a temporal switch in the pattern of leukocyte recruitment seen during acute inflammation. Immunity 2001; 14 : 705-714.
73. Venkatachalam K, Mummidi S, Cortez DM, Prabhu SD, Valente AJ, Chandrasekar B. Resveratrol inhibits high glucoseinduced PI3K/Akt/ERK-dependent interleukin-17 expression in primary mouse cardiac fibroblasts. Am J Physiol Heart Circ Physiol 2008; 294: H2078-H2087.

74. Jones SA. Directing transition from innate to acquired immunity: Defining a role for IL-6. J Immunol 2005; 175: 3463-3468.

75. Weissenbach $M$, Clahsen $T$, Weber $C$ et al. Interleukin- 6 is a direct mediator of T cell migration. Eur J Immunol 2004; 34: 2895-2906.

76. Shi-wen $X$, Stanton LA, Kennedy $L$ et al. CCN2 is necessary for adhesive responses to transforming growth factor-beta1 in embryonic fibroblasts. J Biol Chem 2006; 281: 10715-10726.

77. Qi W, Chen X, Polhill TS et al. TGF-beta1 induces IL-8 and MCP-1 through a connective tissue growth factor-independent pathway. Am J Physiol Renal Physiol 2006; 290: F703-F709.

78. Ikawa $Y, N g P S$, Endo $K$ et al. Neutralizing monoclonal antibody to human connective tissue growth factor ameliorates transforming growth factor-beta-induced mouse fibrosis. J Cell Physiol 2008; 216: 680-687.

79. Raichlin E, Villarraga HR, Chandrasekaran K et al. Cardiac allograft remodeling after heart transplantation is associated with increased graft vasculopathy and mortality. Am J Transplant 2009; 9: 132139.

80. Torre-Amione G. Cardiac allograft hypertrophy: A new target for therapy, a surrogate marker for survival? Am J Transplant 2009; 9: $7-8$.

81. Nishida T, Kubota S, Fukunaga T et al. CTGF/Hcs24, hypertrophic chondrocyte-specific gene product, interacts with perlecan in regulating the proliferation and differentiation of chondrocytes. J Cell Physiol 2003; 196: 265-275.

82. Duisters RF, Tijsen AJ, Schroen B et al. miR-133 and miR-30 regulate connective tissue growth factor: Implications for a role of microRNAs in myocardial matrix remodeling. Circ Res 2009; 104: 170-178, 176p following 178.

83. Liu W, Feng W, Wang F et al. Osteoprotegerin/RANK/RANKL axis in cardiac remodeling due to immuno-inflammatory myocardial disease. Exp Mol Pathol 2008; 84: 213-217.

84. Okada $H$, Kikuta $T$, Inoue $T$ et al. Dexamethasone induces connective tissue growth factor expression in renal tubular epithelial cells in a mouse strain-specific manner. Am J Pathol 2006; 168: 737-747.

85. Holmes A, Abraham DJ, Sa S, Shiwen X, Black CM, Leask A. CTGF and SMADs, maintenance of scleroderma phenotype is independent of SMAD signaling. J Biol Chem 2001; 276: 10594-10601.

86. Yoshimura A, Naka T, Kubo M. SOCS proteins, cytokine signalling and immune regulation. Nat Rev Immunol 2007; 7: 454465. 